\title{
EGFR-TKI therapy for patients with brain metastases from non-small-cell lung cancer: a pooled analysis of published data
}

This article was published in the following Dove Press journal:

OncoTargets and Therapy

10 November 2014

Number of times this article has been viewed

Yun Fan ${ }^{1,2}$

Xiaoling $\mathrm{Xu}^{3}$

Conghua $\mathrm{Xie}^{4}$

'Zhongnan Hospital of Wuhan University, Department of Radiation Oncology, Wuhan, People's Republic of China; ${ }^{2}$ Department of Chemotherapy, Zhejiang Cancer Hospital, Hangzhou, People's Republic of China; ${ }^{3}$ Zhejiang Cancer Hospital, Hangzhou, People's Republic of China; ${ }^{4}$ Zhongnan Hospital of Wuhan University, Department of Radiation Oncology, Wuhan, People's Republic of China
Correspondence: Conghua Xie Zhongnan Hospital of Wuhan University, Department of Radiation Oncology, Wuhan, People's Republic of China 43007I

Tel +86 I36 38607566

Fax +86 57I 88I28208

Email conghuaxx@163.com
Introduction: Brain metastases are one of the leading causes of death from non-small-cell lung cancer (NSCLC). The use of epidermal growth factor receptor (EGFR) tyrosine kinase inhibitors (TKIs) to treat brain metastases remains controversial. Thus, we performed a pooled analysis of published data to evaluate the efficacy of EGFR-TKIs in NSCLC patients with brain metastases, particularly for tumors with activating EGFR mutations.

Methods: Several data sources were searched, including PubMed, Web of Science, and ASCO Annual Meetings databases. The end points were intracranial overall response rate (ORR), disease control rate (DCR), progression-free survival (PFS), overall survival (OS), and adverse events. The pooled ORR, DCR, PFS, and OS with 95\% confidence intervals (CIs) were calculated employing fixed- or random-effect models, depending on the heterogeneity of the included studies.

Results: Sixteen published studies were included in this analysis, with a total of 464 enrolled patients. The EGFR mutational status was unknown for 362 (unselected group), and 102 had activating EGFR mutations. The pooled intracranial ORR and DCR were $51.8 \%$ (95\% CI: 45.8\%-57.8\%) and 75.7\% (95\% CI: 70.3\%-80.5\%), respectively. A higher ORR was observed in the EGFR mutation group than in the unselected group ( $85.0 \%$ vs $45.1 \%$ ); a similar trend was observed for the DCR $(94.6 \%$ vs $71.3 \%)$. The pooled median PFS and OS were 7.4 months (95\% CI, 4.9-9.9) and 11.9 months (95\% CI, 7.7-16.2), respectively, with longer PFS (12.3 months vs 5.9 months) and OS (16.2 months vs 10.3 months) in the EGFR mutation group than in the unselected group.

Conclusion: This pooled analysis strongly suggests that EGFR-TKIs are an effective treatment for NSCLC patients with brain metastases, particularly in those patients harboring EGFR mutations. Larger prospective randomized clinical trials are warranted to confirm our conclusion and identify the most appropriate treatment model.

Keywords: NSCLC, brain metastases, epidermal growth factor receptor, tyrosine kinase inhibitors

\section{Introduction}

Brain metastases are a frequent complication in patients with non-small-cell lung cancer (NSCLC), and approximately $25 \%-40 \%$ of NSCLC patients develop brain metastases during their disease course. ${ }^{1,2}$ Traditionally, whole-brain radiation therapy (WBRT) has been the standard treatment for brain metastases with multiple intracranial lesions; in some cases, surgical resection, stereotactic radiosurgery, or some combination of the three can be used..$^{3-6}$ However, the therapeutic effects are limited and the prognosis remains poor. Median survival ranges from 2.4 months to 4.8 months for patients with 
brain metastases who receive WBRT alone. ${ }^{7,8}$ Because it is assumed that most chemotherapeutic agents cannot cross the blood-brain barrier, the efficacy of these drugs in controlling NSCLC-related brain metastases remains controversial.

Erlotinib and gefitinib, small-molecule tyrosine kinase inhibitors (TKIs) of epidermal growth factor receptor (EGFR), have been shown to improve survival in NSCLC when used as a second-line therapy compared with placebo either for an entire unselected group of NSCLC patients or in certain subgroups, such as never-smokers or patients of Asian origin. ${ }^{9,10}$ Subsequently, activating EGFR mutations were determined to be predictive parameters of the response to EGFR-TKI therapy in NSCLC. ${ }^{11-13}$ EGFR-TKIs are now recognized as a standard first-line therapy replacing conventional cytotoxic chemotherapy for patients with activating $E G F R$ mutations in response to randomized studies that demonstrated significantly higher tumor overall response rates (ORR) and longer progression-free survival (PFS). ${ }^{14-17}$ In contrast, for previously treated patients with wild-type EGFR, compared with first-generation EGFR-TKIs, conventional chemotherapy was associated with improvement in PFS. ${ }^{18-20}$

Evidence suggests that EGFR-TKIs can penetrate the blood-brain barrier. ${ }^{21,22}$ Several case reports and studies with a small series of patients have indicated successful treatment of brain metastases with TKIs. ${ }^{23-27}$ However, the results were not consistent with the cerebral ORR of $10 \%-86 \%$ and PFS of 3-10 months. ${ }^{26,27}$ Most of these studies included small sample sizes, retrospective analyses, and case reports with inadequate power to exclude clinically relevant differences in efficacy. Thus, we performed the present pooled analysis to evaluate the efficacy of EGFR-TKIs in NSCLC patients with brain metastases, particularly in NSCLC patients harboring activating EGFR mutations.

\section{Methods}

\section{Literature search strategy}

The selection of publications for inclusion was performed independently by two authors (Yun Fan and Xiaoling Xu), with the last search performed on December 25, 2013. A computerized search was performed using the PubMed (from 1966 to the present), Web of Science (from 1945 to the present), online proceedings of the ASCO Annual Meetings (from 2007 to the present), EBSCO (from 1975 to the present), MEDLINE (from 1975 to the present), and Springer Link (from 1997 to the present) databases using the following search keywords: "lung cancer", "non-small-cell lung cancer", "brain metastases", "EGFR-TKI", "erlotinib", and "gefitinib".
Manual searches were performed by reviewing the reference lists of the retrieved studies and review articles to identify additional potentially eligible studies.

\section{Study eligibility}

Study selection was based on an initial screening of the identified abstracts or titles and a second screening of the full-text articles. Studies were considered eligible if they met the following criteria: 1) prospective cohorts, retrospective designs, or clinical trials were all included because of the small number of relevant articles; 2) patients with brain metastases from NSCLC were treated with erlotinib or gefitinib; 3) the events of intracranial complete response (CR), partial response (PR), stable disease (SD) or progressive disease (PD), ORR, and disease control rate (DCR) were reported; 4) PFS and overall survival (OS) with corresponding 95\% confidence intervals (CIs) were reported; 5) the number of study cases was greater than five; and 6) the publication was written in English. Research protocol articles, case reports, letters to the editor, reviews, articles based on guidelines, and articles published in books were not included.

\section{Data extraction and quality assessment}

In all identified reports, NSCLC patients with brain metastases were treated with gefitinib or erlotinib. The treatment response was determined by the Response Evaluation Criteria in Solid Tumors (RECIST), ${ }^{28}$ and toxicities were assessed according to the Common Terminology Criteria for Adverse Events (CTCAE) Version 2.0 or 3.0. The following information was extracted from each publication: first author, year of publication, number of patients analyzed, median age or mean age of the population, performance status, type of study, $E G F R$ mutation, former treatments before target therapy, duration of follow-up, adverse reaction, and the events of CR, PR, SD or PD, and PFS or OS, with corresponding 95\% CIs. To extract the data, two of the authors (Yun Fan and Xiaoling $\mathrm{Xu}$ ) independently extracted the information from each eligible publication. Any disagreement was settled by a third investigator (Conghua Xie). No authors of the original publications were contacted for verification or clarification of their data.

\section{Statistical analysis}

ORR was defined as CR plus PR, whereas DCR was defined as the best tumor response of CR plus PR plus SD. The rates of both responses to EGFR-TKI target therapy for brain metastases in NSCLC patients were calculated as the event rate along with the $95 \%$ CI. Toxicity was summarized 
using descriptive statistics. The fixed-effects model (Mantel-Haenszel method) was used for cases with no significant heterogeneity. Otherwise, the random-effects model (DerSimonian and Laird method) was used. The homogeneity of the studies was tested by the $Q$ statistic (significance level at $P>0.10)$ and the $I^{2}$ statistic $\left(I^{2}=0 \%-50 \%\right.$ for no or moderate heterogeneity; $P>50 \%$, significant heterogeneity), which are quantitative measures of inconsistency across studies. ${ }^{29}$

Funnel plots were used to evaluate possible publication bias regarding each study outcome. We conducted subgroup analyses stratified by EGFR mutation status, study type, concurrent WBRT, type of EGFR-TKI used, and histology type to assess the impact of these variables on outcomes. We also conducted a sensitivity analysis to investigate the influence of a single study on the overall risk estimate by omitting one study in turn. All tests were two-sided, and a $P$-value less than 0.05 was considered significant. All statistical analyses, including the combined ORR, DCR, and median pooled PFS and OS, were calculated using Meta-Analyst software ${ }^{30}$ (Version Beta 3.13; Tufts Evidence-based Practice Center, Boston, MA, USA) using the one-arm binary or continuous analysis function.

\section{Results}

\section{Literature search}

A flow chart showing the studies involved is presented in Figure 1. Briefly, we identified 151 records by searching the specified databases, and 30 records were identified through other sources, such as the references of published articles and conference articles. After removing duplicate records, 120 records remained. Further screening revealed that

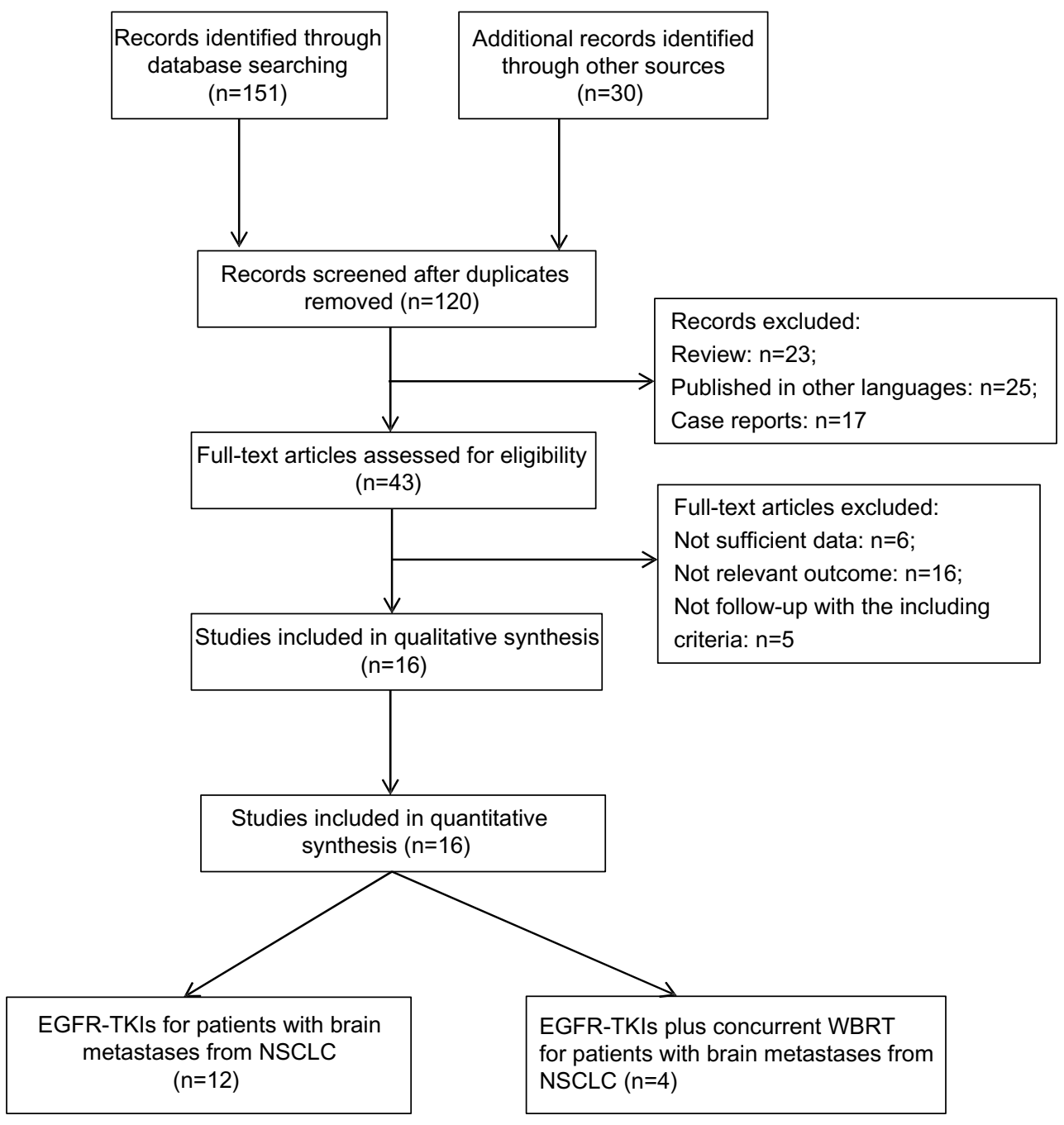

Figure I Selection of publications included in the pooled analysis.

Abbreviations: EGFR-TKIs, small-molecule tyrosine kinase inhibitors of epidermal growth factor receptor; NSCLC, non-small-cell lung cancer; WBRT, whole-brain radiation therapy. 
43 potential studies were full-text articles. After eliminating articles that did not satisfy the selection criteria, did not permit the extraction of sufficient data, or were not relevant, 16 studies $^{23-27,31-41}$ published between 2003 and 2013 were finally selected that met the qualitative and quantitative requirements of the systematic analysis.

\section{Characteristics of the studies}

Of the 16 included articles, six were Phase II single-arm trials, one was a Phase I single-arm trial, five were prospective in design, and four were retrospective analyses. Four hundred sixty-four patients were enrolled in this pooled analysis. Among them, approximately half of the patients were from East Asia, including China, Japan, and Korea, while the others were from Western countries and the US. The median age of the patients ranged from 54 years to 65.5 years. The patients of most studies had a performance status of $0-3$, and the median length of follow-up ranged from 2.6 months to 28.5 months. In addition, the patients in most of the unselected studies had received prior chemotherapy or radiotherapy.

All patients were treated with oral gefitinib or erlotinib and were followed until disease progression, death, or intolerable side effects. Of the 464 enrolled patients, the EGFR mutational status was unknown for 362 (unselected group), and 102 harbored activating EGFR mutations. The ORR for EGFR-TKI treatment was available for
428 patients: 358 patients with brain metastases from NSCLC were treated with EGFR-TKI only, and the other 70 patients were treated with EGFR-TKIs plus concurrent WBRT. PFS and OS were available in eight and ten studies, respectively. Two reports ${ }^{7,11}$ only included patients with EGFR mutations, and three other studies ${ }^{9,15,21}$ provided data for extracting ORR, DCR, PFS, and OS in patients with and without EGFR mutations. The remaining studies did not provide patients with the EGFR mutation status. The details of the patient demographics and study designs are included in Table 1.

\section{Main analysis}

Based on the search strategy, 16 eligible studies were identified and included in the pooled analysis for ORR and DCR. Due to the modest significant heterogeneity of ORR ( $Q$ statistic, $\left.0.991 ; I^{2}, 0.468\right)$ and DCR ( $Q$ statistic, 0.986; $\left.I^{2}, 0.447\right)$, the fixed-effects model was used to analyze the data. The pooled ORR was 51.8\% (95\% CI: 45.8\%-57.8\%, Figure 2A), and the pooled DCR was $75.7 \%$ (95\% CI: $70.3 \%-80.5 \%$, Figure 2B). By contrast, significant heterogeneity $\left(I^{2}>50 \%\right)$ was observed in both PFS $(7.4 ; 95 \%$ CI: 4.9-9.9; Figure 2C) and OS (11.9; 95\% CI: 7.7-16.2; Figure 2D), and thus the random-effects model was used. The weighted overall median PFS and survival time were 7.4 months (95\% CI: 7.7-16.2; Figure 2C) and 11.9 months (95\% CI: 4.9-9.9; Figure 2D), respectively.

Table I Characteristics of the studies

\begin{tabular}{|c|c|c|c|c|c|c|c|c|c|}
\hline Authors & Type of study & $\begin{array}{l}\text { EGFR } \\
\text { mutation (+) } \\
\text { status }\end{array}$ & $\begin{array}{l}\text { Whether } \\
\text { concurrent } \\
\text { WBRT }\end{array}$ & $\begin{array}{l}\text { Number } \\
\text { of patients }\end{array}$ & $\begin{array}{l}\text { The type of } \\
\text { EGFR-TKI }\end{array}$ & ORR & DCR & $\begin{array}{l}\text { PFS, } \\
\text { months }\end{array}$ & $\begin{array}{l}\text { OS, } \\
\text { months }\end{array}$ \\
\hline Bai and $\mathrm{Han}^{26}$ & Retrospective & Part of patients & No & 40 & Erlotinib & $10.00 \%$ & $62.50 \%$ & 3 & 9.2 \\
\hline Ceresoli et $\mathrm{al}^{24}$ & Prospective & Unselected & No & 41 & Gefitinib & $9.80 \%$ & $26.80 \%$ & 3 & 5 \\
\hline Chiu et $\mathrm{al}^{36}$ & Prospective & Unselected & No & 21 & Gefitinib & $50.00 \%$ & $90.48 \%$ & NA & NA \\
\hline Hotta et $\mathrm{al}^{35}$ & Retrospective & Unselected & No & 14 & Gefitinib & $42.86 \%$ & $100.00 \%$ & 8.8 & 9.1 \\
\hline luchi et $\mathrm{a}^{27}$ & Phase II & All the patients & No & 41 & Gefitinib & $87.80 \%$ & $97.56 \%$ & 14.5 & 21.9 \\
\hline Kim et $\mathrm{al}^{37}$ & Prospective & Unselected & No & 23 & $\begin{array}{l}\text { Gefitinib or } \\
\text { erlotinib }\end{array}$ & $73.90 \%$ & $82.60 \%$ & 7.1 & 18.8 \\
\hline Lind et $\mathrm{al}^{40}$ & Phase I & Unselected & Yes & 7 & Erlotinib & $71.43 \%$ & $100.00 \%$ & NA & 4.7 \\
\hline Ma et $\mathrm{al}^{39}$ & Phase II & Unselected & Yes & 21 & Gefitinib & $80.95 \%$ & $95.24 \%$ & 10 & 13 \\
\hline Namba et $\mathrm{a}^{23}$ & Prospective & Unselected & No & 11 & Gefitinib & $81.82 \%$ & $100.00 \%$ & 2.4 & 12 \\
\hline Olmez et $\mathrm{al}^{41}$ & Retrospective & Unselected & Yes & 8 & Erlotinib & $33.33 \%$ & $100.00 \%$ & NA & $\mathrm{I} .4$ \\
\hline Park et al ${ }^{34}$ & Phase II & All the patients & No & 27 & $\begin{array}{l}\text { Gefitinib or } \\
\text { erlotinib }\end{array}$ & $85.19 \%$ & $96.30 \%$ & 6.6 & 15.9 \\
\hline Porta et $\mathrm{al}^{38}$ & Retrospective & Part of patients & No & 53 & Erlotinib & $26.42 \%$ & $84.91 \%$ & 2.9 & 4.3 \\
\hline Rosell et a ${ }^{32}$ & Prospective & All the patients & No & 30 & Erlotinib & NR & $N R$ & 10 & 18 \\
\hline Welsh et $\mathrm{a}^{25}$ & Phase II & Part of patients & Yes & 40 & Erlotinib & $91.67 \%$ & $94.44 \%$ & 8 & 11.8 \\
\hline Wu et $\mathrm{a}^{31}$ & Phase II & Unselected & No & 40 & Gefitinib & $37.50 \%$ & $82.50 \%$ & 9 & 15 \\
\hline Wu et $\mathrm{al}^{33}$ & Phase II & Part of patients & No & 47 & Erlotinib & $59.57 \%$ & $76.60 \%$ & 10.1 & 18.9 \\
\hline
\end{tabular}

Abbreviations: DCR, disease control rate; EGFR, epidermal growth factor receptor; NA, not available; NR, not reported; ORR, overall response rate; OS, overall survival; PFS, progression-free survival; TKI, tyrosine kinase inhibitor; WBRT, whole-brain radiation therapy. 

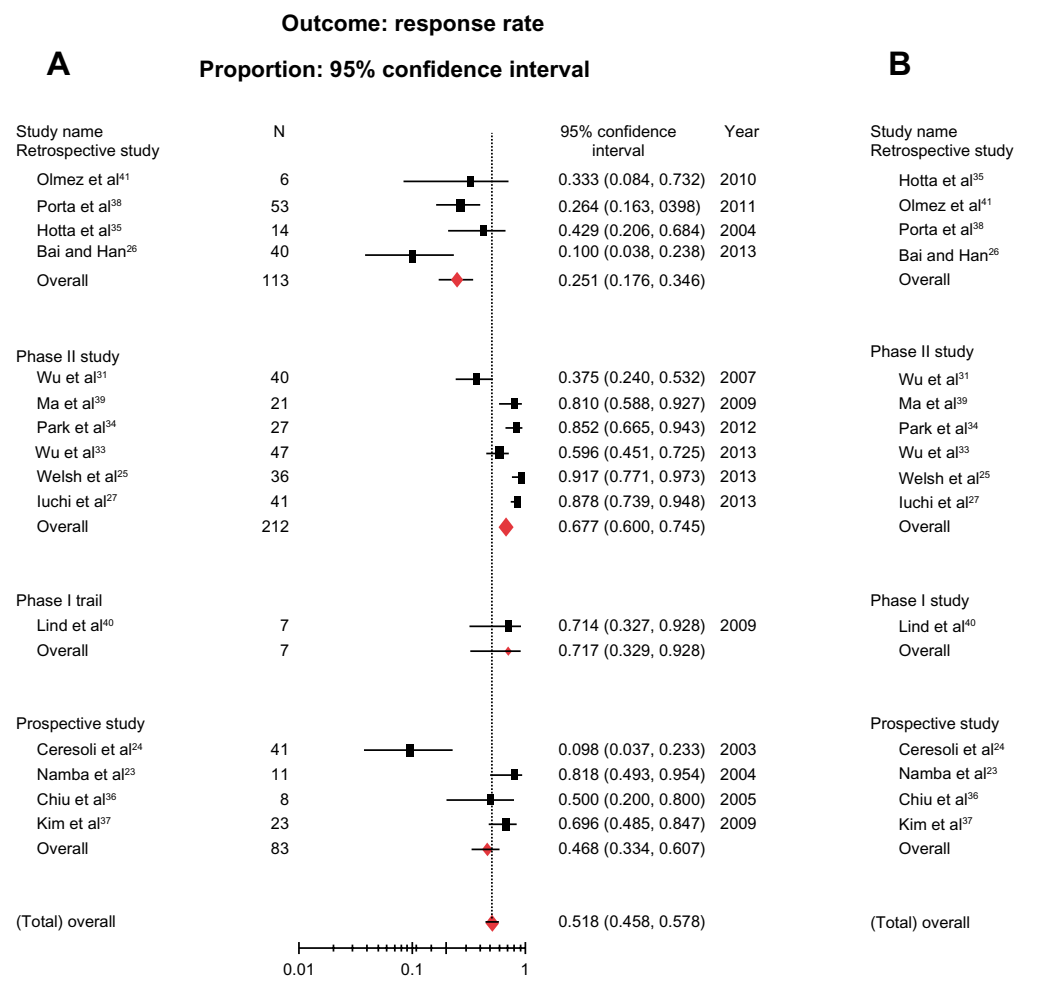

Outcome: progression free survival

Forest plot: $95 \%$ confidence interval

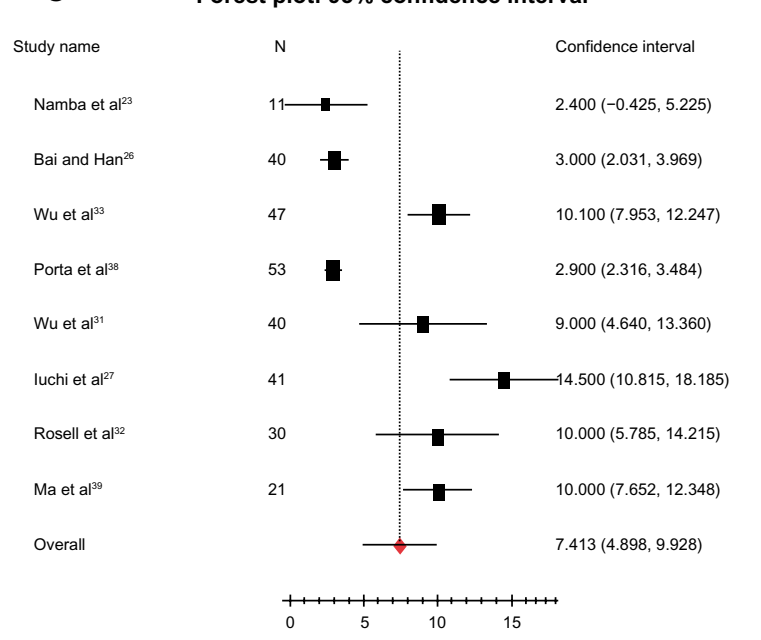

B

Outcome: disease control rate

Proportion: $95 \%$ confidence interval

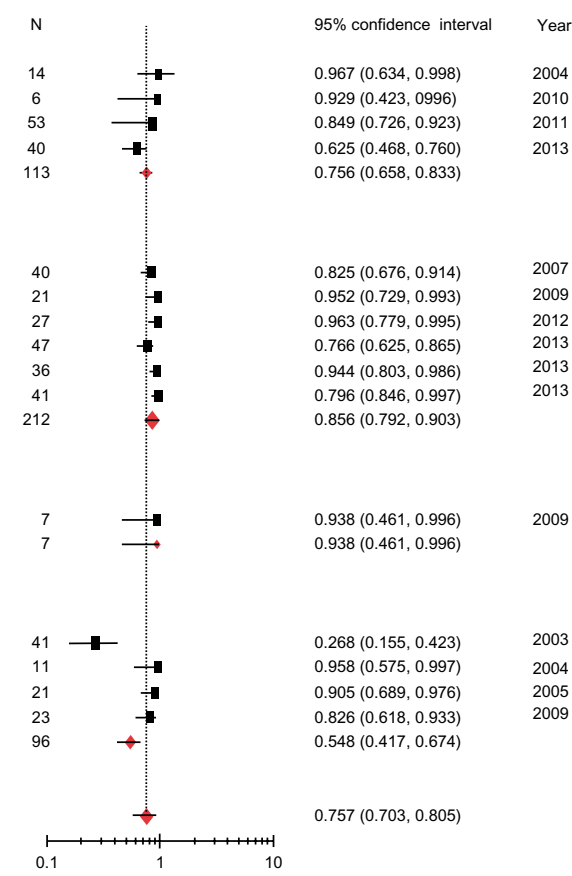

D

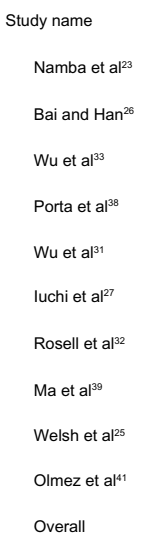

Outcome: overall survival

Forest plot: $95 \%$ confidence interval

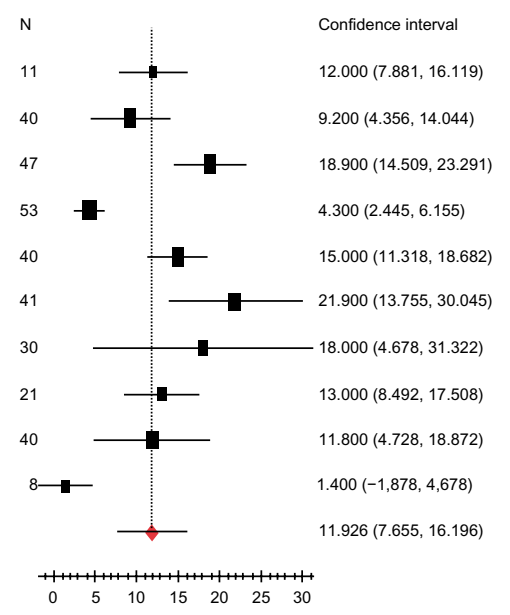

Figure 2 Results of main analysis.

Notes: (A) Pooled analysis for response rate. (B) Pooled analysis for disease control rate. (C) Pooled analysis for progression-free survival. (D) Pooled analysis for overall survival.

Side effects were generally mild and consisted mainly of diarrhea, skin toxicity, and liver dysfunction, with respective incidences of $0 \%-60.0 \%, 7.4 \%-100 \%$, and $0 \%-18.2 \%$ (Table 1).

\section{Subgroup and sensitivity analyses}

The high degree of heterogeneity between the studies warranted explanation. Therefore, subgroup analysis was performed. Table 2 shows the results of subgroup analyses stratified by EGFR mutation status, study type, concurrent WBRT, the type of EGFR-TKI used, and histology type.

In the subgroup analysis, among patients with brain metastases from NSCLC who harbored EGFR mutations, fine homogeneity was observed in the combined data for the pooled ORR ( $Q$ statistic, $\left.0.514 ; I^{2}, 0.000\right)$, DCR $(Q$ statistic, $\left.0.700 ; I^{2}, 0.000\right)$, PFS $\left(I^{2}, 0.000\right)$, and OS $\left(I^{2}, 0.200\right)$. The ORR (Figure 3A) was higher in the EGFR mutation group $(85.0 \%, 95 \%$ CI: $76.5 \%-90.7 \%)$ than in the unselected 
Table 2 Results from subgroup analysis

\begin{tabular}{|c|c|c|c|c|}
\hline Subgroup & $\begin{array}{l}\text { Pooled ORR } \\
\text { and } 95 \% \mathrm{Cl} \text {, model }\end{array}$ & $\begin{array}{l}\text { Pooled DCR } \\
\text { and } 95 \% \mathrm{Cl} \text {, model }\end{array}$ & $\begin{array}{l}\text { Pooled PFS (months) } \\
\text { and } 95 \% \mathrm{Cl} \text {, model }\end{array}$ & $\begin{array}{l}\text { Pooled OS (months) } \\
\text { and } 95 \% \mathrm{Cl} \text {, model }\end{array}$ \\
\hline \multicolumn{5}{|l|}{ Histology } \\
\hline Adenocarcinoma & $0.604(0.493-0.706)$, fixed & $0.728(0.662-0.784)$, fixed & NA & NA \\
\hline Unselected & $0.484(0.415-0.555)$, fixed & $0.728(0.662-0.784)$, fixed & & \\
\hline \multicolumn{5}{|l|}{ Study design } \\
\hline Retrospective study & $0.25 I(0.176-0.346)$, fixed & $0.756(0.658-0.833)$, fixed & NA & NA \\
\hline Prospective study & $0.468(0.334-0.607)$, fixed & $0.417(0.548-0.674)$, fixed & NA & NA \\
\hline Phase II study & $0.677(0.600-0.745)$, fixed & $0.856(0.792-0.903)$, fixed & $10.573(9.192-11.953)$, fixed & I5.685 (13.503-17.868), fixed \\
\hline \multicolumn{5}{|l|}{ EGFR mutation status } \\
\hline Mutation & $0.850(0.765-0.907)$, fixed & $0.946(0.87 I-0.979)$, fixed & I2.334 (I0.4| I-|4.257), fixed & $16.169(|2.26|-20.076)$, fixed \\
\hline Unselected & 0.45 I (0.376-0.528), fixed & $0.713(0.650-0.768)$, fixed & 5.937 (3.5 I8-8.356), random & $10.318(5.013-15.623)$, random \\
\hline \multicolumn{5}{|c|}{ Whether concurrent WBRT } \\
\hline Yes & $0.662(0.79 \mid-0.880)$, fixed & $0.944(0.860-0.979)$, fixed & NA & NA \\
\hline No & 0.47 I (0.408-0.535), fixed & $0.73 \mathrm{I}(0.67 \mathrm{I}-0.784)$, fixed & 6.965 (4.450-9.480), random & I 3.565 (8.104-19.025), random \\
\hline \multicolumn{5}{|l|}{ The type of EGFR-TKI } \\
\hline Gefitinib & $0.518(0.426-0.609)$, fixed & $0.687(0.582-0.776)$, fixed & 8.903 (3.909-13.897), random & |4.13| (II.878-16.384), fixed \\
\hline Erlotinib & $0.443(0.358-0.53 \mathrm{I})$, fixed & $0.778(0.706-0.836)$, fixed & 5.937 (3.270-8.607), random & 9.714 (4.168-I5.259), random \\
\hline
\end{tabular}

Abbreviations: $\mathrm{Cl}$, confidence interval; DCR, disease control rate; EGFR, epidermal growth factor receptor; NA, not available; ORR, overall response rate; OS, overall survival; PFS, progression-free survival; TKI, tyrosine kinase inhibitor; WBRT, whole-brain radiation therapy.

group (unknown EGFR mutational status) (45.1\%, 95\% CI: $37.6 \%-52.8 \%$ ), and a similar trend was observed for the DCR (Figure 3B) $(94.6 \%, 95 \%$ CI: 87.1\%-97.9\% vs 71.3\%, 95\% CI: $65.0 \%-76.8 \%$, respectively). The pooled PFS (Figure 3C) was longer in the EGFR mutation group than in the unselected group: 12.3 months (95\% CI: 10.4-14.3) versus 5.9 months (95\% CI: 3.5-8.4), with no evidence of heterogeneity in the EGFR mutation group $\left(P=0.525, I^{2}=0.000\right)$. Similarly, the pooled OS (Figure 3D) was longer in the EGFR mutation group than in the unselected group: 16.2 months versus 10.3 months, respectively.

In addition, no significant heterogeneity was observed in the Phase II studies $\left(I^{2}<0.50\right)$. The Phase II studies reported higher ORR (Figure 2A) and DCR (Figure 2B) rates and more favorable survival (Table 2). EGFRTKIs plus concurrent WBRT yielded a higher ORR (66.2\% vs $47.1 \%$ ) and DCR (94.4\% vs $73.1 \%$ ) than treatment with EGFR-TKIs alone. Moreover, patients with brain metastases from NSCLC treated with gefitinib had improved survival compared with those treated with erlotinib (14.1 months vs 9.7 months).

\section{Sensitivity analyses and publication bias}

The sensitivity analyses demonstrated that no individual study affected the ORR, DCR, PFS, and OS, because omission of any single study did not significantly affect these parameters. No evidence of publication bias was found with regard to ORR, DCR, PFS, and OS in relation to EGFR-TKI treatment for patients with brain metastases from NSCLC, as suggested by the funnel plot. In addition, no significant publication bias was identified in the EGFR mutation group (Figure 4) and Phase II studies.

\section{Discussion}

The present pooled analysis included 464 patients from 16 trials; approximately half of the patients were from East Asia, while the others were from Western countries and the US. The primary objective was to evaluate the value of EGFRTKI therapy in patients with brain metastases from NSCLC. We believe that our pooled analysis strengthens the individual observations of each of these small prospective and retrospective studies. The results confirmed that in this population of patients, EGFR-TKIs yield significant beneficial effects, with a pooled intracranial ORR of $51.8 \%$, DCR of $75.7 \%$, median PFS of 7.4 months, and OS of 11.9 months. These results were associated with a longer OS than with WBRT alone. ${ }^{7,8}$ At least two reasons for the therapeutic efficacy of EGFR-TKIs in NSCLC patients with brain metastases can be proposed. First, EGFR-TKIs might penetrate the brain-blood barrier to reach the intracranial lesion; the documented data revealed penetration rates of $1.13 \% \pm 0.36 \%$ and $2.77 \% \pm 0.45 \%$ for gefitinib and erlotinib, respectively. ${ }^{21,22,42}$ Second, a considerable proportion of patients with brain metastases also have extracranial lesions and require systemic medication. Thus, EGFR-TKIs represent an effective therapy in advanced NSCLC.

The EGFR mutation status seems to be the strongest correlate of the response to EGFR-TKIs. ${ }^{11-13}$ However, EGFR mutations in the human population are apparently heterogeneous. For instance, Paez et $\mathrm{al}^{12}$ reported mutation differences 
A

Outcome: response rate

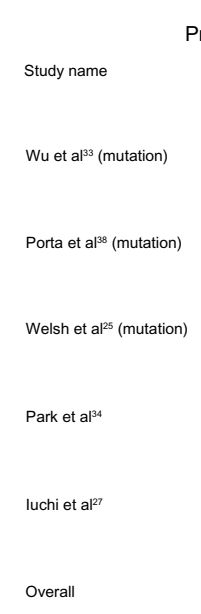

\section{C}

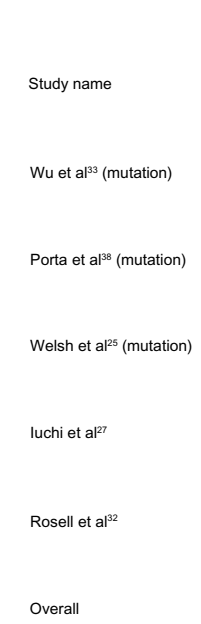

Proportion: 95\% confidence interval

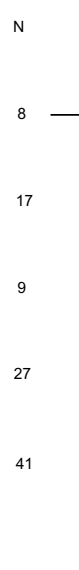

Forest plot: 95\% confidence interval

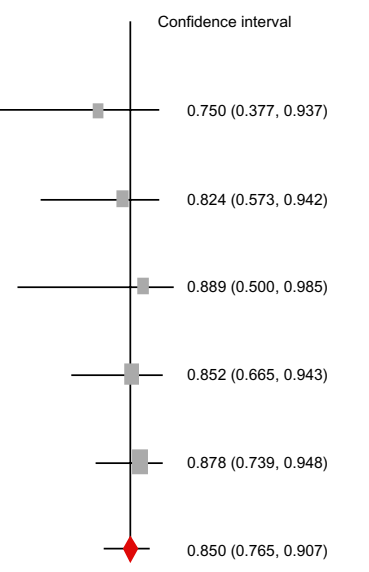

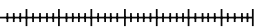
$\begin{array}{llllllll}0.4 & 0.5 & 0.6 & 0.7 & 0.8 & 0.9 & 1.0 & 1.1\end{array}$

Outcome: progression free survival

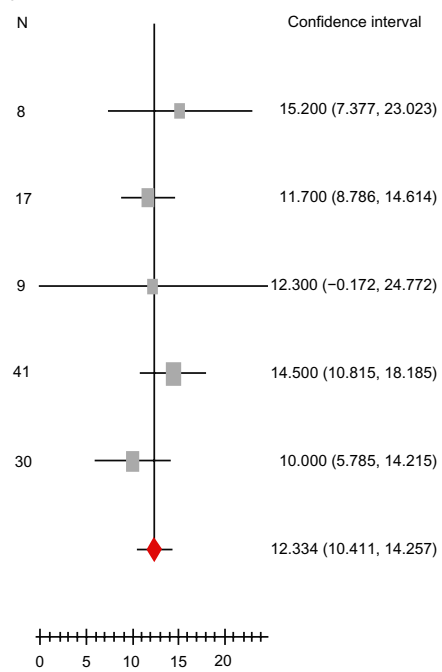

B

Outcome: disease control rate

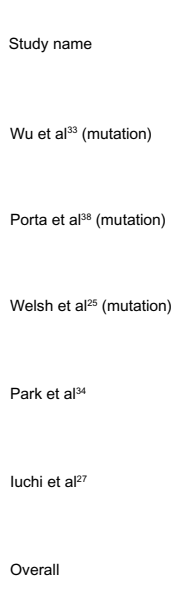

Proportion: 95\% confidence interval

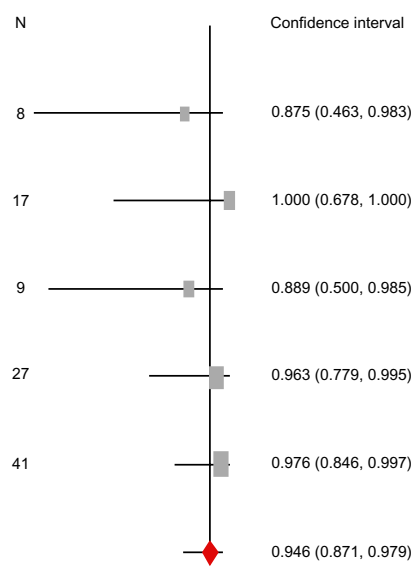

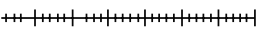
$\begin{array}{lllllll}0.5 & 0.6 & 0.7 & 0.8 & 0.9 & 1.0 & 1.1\end{array}$ Outcome: overall survival

D

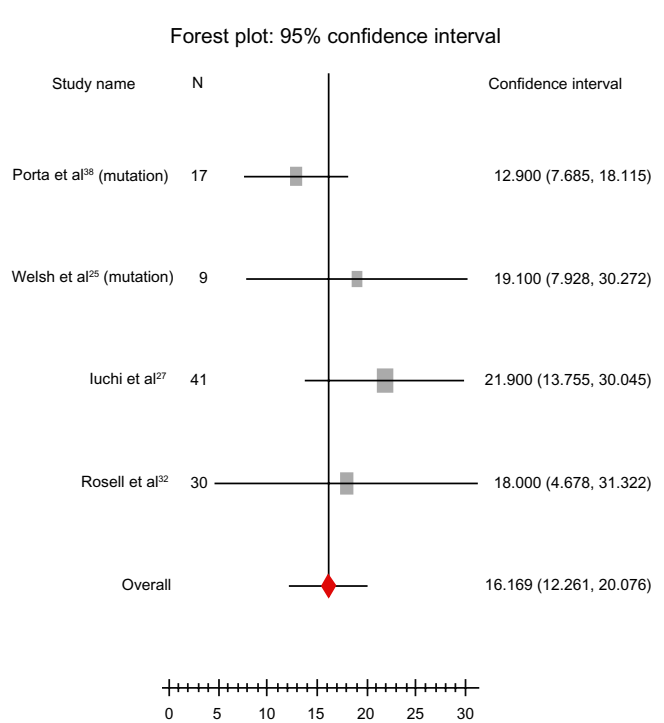

Figure 3 Results of subgroup analyses.

Notes: (A) Pooled analysis for response rate in the epidermal growth factor receptor (EGFR) mutation group. (B) Pooled analysis for disease control rate in the EGFR mutation group. (C) Pooled analysis for progression-free survival in the EGFR mutation group. (D) Pooled analysis for overall survival in the EGFR mutation group.

according to sex $(20 \%$ vs $9 \%$ for females and males, respectively), tumor histology ( $21 \%$ vs $2 \%$ for adenocarcinoma and other types, respectively), and ethnicity ( $26 \%$ vs $2 \%$ for Asian and North American populations, respectively). Recently, Shi et al ${ }^{43}$ tested 1,482 NSCLC patients with adenocarcinoma from seven Asian regions and found an overall rate of EGFR mutations of $51.4 \%$. However, the rate was $15.0 \%$ in similar patients in the US. ${ }^{44}$ Thus, the efficacy of EGFR-TKI treatment may depend on the treated population. Therefore, to exclude possible confounding factors, we conducted subgroup analysis by dividing the patients into an EGFR mutation group and an unselected group (unknown EGFR mutational status). Our data demonstrated that, compared with the unselected group, therapeutic benefits are observed in patients with $E G F R$ mutations with an ORR of $85.0 \%$ versus $45.1 \%$ and a DCR of $94.6 \%$ versus $71.3 \%$ for the $E G F R$ mutation and unselected groups, respectively. EGFR mutations also showed therapeutic advantage over unselected groups in terms of median progression-free survival (12.3 months vs 5.9 months) and median survival time (16.2 months vs 10.3 months), indicating that EGFR-TKIs may be suitable for the treatment of brain metastases in patients with $E G F R$ 


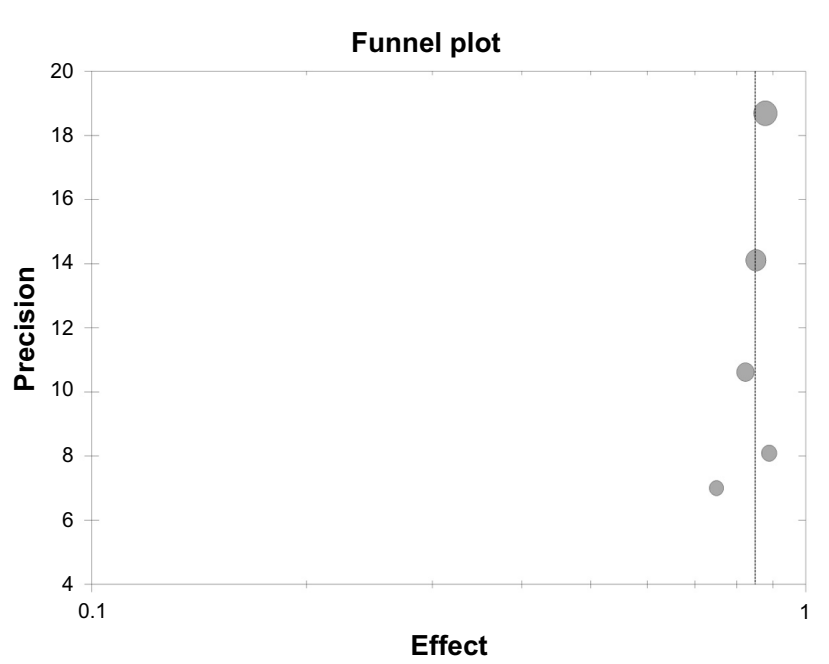

Figure 4 Funnel plot for studies of epidermal growth factor receptor mutation patients.

mutations. Currently, EGFR-TKIs are approved for use as a first-line therapy for patients with EGFR-mutated lung cancer, and it seems reasonable to consider EGFR-TKIs as a first-line treatment for EGFR mutations with brain metastases. Further randomized clinical trials are warranted.

Of the 16 pooled studies, 358 patients in 12 studies were administered EGFR-TKIs alone, while 70 patients in four studies were concurrently treated with WBRT and EGFR-TKIs. Subgroup analysis indicated that concurrent administration of WBRT and EGFR-TKIs might be more beneficial than administration of EGFR-TKIs alone in unselected patients, with an ORR of $66.2 \%$ versus $45.2 \%$ and a DCR of $94.4 \%$ versus $73.1 \%$, respectively. Because no survival data were reported in the two studies of the concurrent treatment groups, we could not infer whether the enhanced ORR and DCR for the concurrent treatment groups suggested a survival advantage. Although blockade of $E G F R$ signaling in vitro has been shown to sensitize cells to the effects of radiation, ${ }^{45}$ this effect has not been confirmed in clinical studies. EGFR-TKIs showed high rates of remission in EGFR mutant patients with brain metastases, and concurrent administration of WBRT and EGFR-TKIs among these patients is being disputed. Recent data by Iuchi et $\mathrm{al}^{27}$ indicated that the adequate effect of gefitinib could withhold radiation therapy safety to avoid neurocognitive deterioration in patients with EGFR mutations and brain metastases. The ability of EGFR-TKIs to replace WBRT as a first-line treatment is under investigation (NCT01724801) in a stage III randomized clinical trial aimed to investigate whether icotinib enhances PFS compared with WBRT in patients with $E G F R$ mutations and brain metastases. ${ }^{46}$
Finally, subgroup analysis based on different EGFR-TKI agents indicated that gefitinib resulted in improved survival compared with erlotinib, with a PFS of 8.9 months versus 5.9 months and OS of 14.1 months versus 9.7 months. The different outcomes of erlotinib and gefitinib treatment may be due to the following. First, because the frequency of EGFR mutations is higher in Asian than in Caucasian populations, EGFR-TKIs may have had better efficacy in Asian patients in the unselected group. In our data, clinical studies with gefitinib were mainly conducted in the Asian population, while those with erlotinib were not. Second, bias may be induced by smallsized and nonrandomized studies. Case reports have shown that erlotinib shows a benefit in gefitinib failure patients with brain metastases, ${ }^{47,48}$ and some investigations indicate that the rate of penetration of the brain barrier by erlotinib is higher than that for gefitinib. ${ }^{42}$ Thus, it is tempting to conclude that erlotinib is more efficacious than gefitinib. However, our pooled analysis does not support this conclusion. Further studies are needed to compare different EGFR-TKI agents in these patients.

The present pooled analysis has some limitations. First, the random-effects model used in part of the analysis may weaken the effect of a large sample with better quality and increase the effect of a small sample with worse quality. Second, because the review was limited to the published literature, and individual participant data are the gold standard for conducting meta-analyses, a potential impact of publication bias could not be ruled out. Third, the number of enrolled cases in some of the studies was relatively small because research focused on a specified patient population is rare. Fourth, heterogeneity might occur due to differences in the treatments that the patients in most of the unselected studies received before EGFR-TKI treatment. Finally, all of the studies were single-arm studies without randomization and blinding, and the comparability was poor. Thus, further studies, particularly prospective randomized control trials, are needed to provide sufficient data for in-depth evaluation. All of these limitations likely affected the final results.

\section{Conclusion}

In conclusion, our pooled analysis demonstrated that EGFRTKIs are an effective treatment for NSCLC patients with brain metastases, particularly in the subgroup with activating EGFR mutations. Therefore, we emphasize that patients with NSCLC should be tested for EGFR mutations. The combination of EGFR-TKIs with WBRT might improve intracerebral ORR and DCR for unselected patients, but the effect of combined treatment in patients with activating EGFR mutations is unclear. Larger prospective randomized clinical trials are 
warranted to confirm the efficacy of EGFR-TKIs alone in patients with activating EGFR mutations.

\section{Disclosure}

The authors report no conflicts of interest in this work.

\section{References}

1. Mujoomdar A, Austin JH, Malhotra R, et al. Clinical predictors of metastatic disease to the brain from non-small cell lung carcinoma: primary tumor size, cell type, and lymph node metastases. Radiology. 2007;242:882-888.

2. Barnholtz-Sloan JS, Sloan AE, Davis FG, et al. Incidence proportions of brain metastases in patients diagnosed (1973 to 2001) in the Metropolitan Detroit Cancer Surveillance System. J Clin Oncol. 2004;22:2865-2872.

3. Tsao MN, Rades K, Wirth A, et al. Radiotherapeutic and surgical management for newly diagnosed brain metastases(es): an American Society for Radiation Oncology evidence-based guideline. Pract Radiat Oncol. 2012;2:210-225.

4. Kocher M, Soffietti R, Abacioglu U, et al. Adjuvant whole-brain radiotherapy versus observation after radiosurgery or surgical resection of one to three cerebral metastases: results of the EORTC 22952-26001 study. J Clin Oncol. 2011;29:134-141.

5. Patchell RA, Tibbs PA, Walsh JW, et al. A randomized trial of surgery in the treatment of single metastases to the brain. $N$ Engl J Med. 1990;22(322):494-500.

6. Andrews DW, Scott CB, Sperduto PW, et al. Whole brain radiation therapy with or without stereotactic radiosurgery boost for patients with one to three brain metastases: Phase III results of the RTOG 9508 randomised trial. Lancet. 2004;363:1665-1672.

7. Knisely JP, Berkey B, Chakravarti A, et al. A phase III study of conventional radiation therapy plus thalidomide versus conventional radiation therapy for multiple brain metastases (RTOG 0118). Int J Radiat Oncol Biol Phys. 2008;71:79-86.

8. Khuntia D, Brown P, Li J, et al. Whole-brain radiotherapy in the management of brain metastases. J Clin Oncol. 2006;24(8):1295-1304.

9. Shepherd FA, Pereira JR, Ciuleanu T, et al. Erlotinib in previously treated non-small-cell lung cancer. $N$ Engl J Med. 2005;353(2):123-132.

10. Thatcher N, Chang A, Parikh P, et al. Gefitinib plus best supportive care in previously treated patients with refractory advanced nonsmall-cell lung cancer: results from a randomised, placebo-controlled, multicentre study (Iressa Survival Evaluation in Lung Cancer). Lancet. 2005;366(9496):1527-1537.

11. Lynch TJ, Bell DW, Sordella R, et al. Activating mutations in the epidermal growth factor receptor underlying responsiveness of non-small-cell lung cancer to gefitinib. N Engl J Med. 2004;350(21):2129-2139.

12. Paez JG, Jänne PA, Lee JC, et al. EGFR mutations in lung cancer: correlation with clinical response to gefitinib therapy. Science. 2004;304(5676):1497-1500.

13. Mok TS, WuYL, Thongprasert S, et al. Gefitinib or carboplatin-paclitaxel in pulmonary adenocarcinoma. N Engl J Med. 2009;361:947-957.

14. Mitsudomi T, Morita S, Yatabe Y, et al. Gefitinib versus cisplatin plus docetaxel in patients with non-small-cell lung cancer harbouring mutations of the epidermal growth factor receptor (WJTOG3405): an open label, randomised phase 3 trial. Lancet Oncol. 2010;11(2):121-128.

15. Zhou C, Wu YL, Chen G, et al. Erlotinib versus chemotherapy as first-line treatment for patients with advanced EGFR mutationpositive non-small-cell lung cancer (OPTIMAL, CTONG-0802): a multicentre, open-label, randomised, phase 3 study. Lancet Oncol. 2011;12(8):735-742.

16. Rosell R, Carcereny E, Gervais R, et al. Erlotinib versus standard chemotherapy as first-line treatment for European patients with advanced EGFR mutation-positive non-small-cell lung cancer (EURTAC): a multicentre, open-label, randomized phase 3 trial. Lancet Oncol. 2012;13:239-246.
17. Wu YL, Zhou C, Hu CP, et al. Afatinib versus cisplatin plus gemcitabine for first-line treatment of Asian patients with advanced non-small-cell lung cancer harbouring EGFR mutations (LUX-Lung 6): an open-label, randomised phase 3 trial. Lancet Oncol. 2014;15(2):213-222.

18. Garassino MC, Martelli O, Broggini M, Farini G, Veronese S, Rulli E, et al. Erlotinib versus docetaxel as second-line treatment of patients with advanced non-small-cell lung cancer and wild-type EGFR tumours (TAILOR): a randomised controlled trial. Lancet Oncol. 2013;14: 981-988.

19. Kawaguchi T, Ando M, Asami K, et al. Randomized phase III trial of erlotinib versus docetaxel as second- or third-line therapy in patients with advanced non-small-cell lung cancer: Docetaxel and Erlotinib Lung Cancer Trial (DELTA). J Clin Oncol. 2014;32(18):1902-1908.

20. Yang J, Cheng Y, Zhao M, et al. A phase II trial comparing pemetrexed with gefitinib as the second-line treatment of nonsquamous NSCLC patients with wild-type EGFR (CTONG-0806). Paper presented at: 2013 ASCO Annual Meeting; May 31 to June 4, 2013; Chicago, IL, USA. Abstract 8042.

21. Broniscer A, Panetta JC, O'Shaughnessy M, et al. Plasma and cerebrospinal fluid pharmacokinetics of erlotinib and its active metabolite OSI-420. Clin Cancer Res. 2007;13(5):1511-1515.

22. Zhao J, Chen M, Zhong W, et al. Cerebrospinal fluid concentrations of gefitinib in patients with lung adenocarcinoma. Clin Lung Cancer. 2013;14(2):188-193.

23. Namba Y, Kijima T, Yokota S, et al. Gefitinib in patients with brain metastases from non-small-cell lung cancer: review of 15 clinical cases. Clin Lung Cancer. 2004;6(2):123-128.

24. Ceresoli GL, Cappuzzo F, Gregorc V, et al. Gefitinib in patients with brain metastases from non-small-cell lung cancer: a prospective trial. Ann Oncol. 2004;15(7):1042-1047.

25. Welsh JW, Komaki R, Amini A, et al. Phase II trial of erlotinib plus concurrent whole-brain radiation therapy for patients with brain metastases from non-small-cell lung cancer. J Clin Oncol. 2013;31(7):895-902.

26. Bai H, Han B. The effectiveness of erlotinib against brain metastases in non-small cell lung cancer patients. Am J Clin Oncol. 2013;36(2): $110-115$.

27. Iuchi T, Shingyoji M, Sakaida T, et al. Phase II trial of gefitinib alone without radiation therapy for Japanese patients with brain metastases from EGFR-mutant lung adenocarcinoma. Lung Cancer. 2013;82(2): 282-287.

28. Therasse P, Arbuck SG, Eisenhauer EA, et al. New guidelines to evaluate the response to treatment in solid tumors. European Organization for Research and Treatment of Cancer, National Cancer Institute of the United States, National Cancer Institute of Canada. J Natl Cancer Inst. 2000;92(3):205-216.

29. Higgins JP, Thompson SG, Deeks JJ, et al. Measuring inconsistency in meta-analyses. BMJ. 2003;327(7414):557-560.

30. Wallace BC, Schmid CH, Lau J, et al. Meta-Analyst: software for pooled-analysis of binary, continuous and diagnostic data. BMC Med Res Methodol. 2009;9:80.

31. Wu C, Li YL, Wang ZM, et al. Gefitinib as palliative therapy for lung adenocarcinoma metastatic to the brain. Lung Cancer. 2007;57(3):359-364.

32. Rosell R, Moran T, Queralt C, et al. Screening for epidermal growth factor receptor mutations in lung cancer. N Engl J Med. 2009;361(10): 958-967.

33. Wu YL, Zhou C, Cheng Y, et al. Erlotinib as second-line treatment in patients with advanced non-small-cell lung cancer and asymptomatic brain metastases: a phase II study (CTONG-0803). Ann Oncol. 2013;24(4):993-999.

34. Park SJ, Kim HT, Lee DH, et al. Efficacy of epidermal growth factor receptor tyrosine kinase inhibitors for brain metastases in non-small cell lung cancer patients harboring either exon 19 or 21 mutation. Lung Cancer. 2012,77(3):556-560.

35. Hotta K, Kiura K, Ueoka H, et al. Effect of gefitinib ('Iressa', ZD1839) on brain metastases in patients with advanced non-small-cell lung cancer. Lung Cancer. 2004;46(2):255-261. 
36. Chiu CH, Tsai CM, Chen YM, et al. Gefitinib is active in patients with brain metastases from non-small cell lung cancer and response is related to skin toxicity. Lung Cancer. 2005;47(1):129-138.

37. Kim JE, Lee DH, Choi Y, et al. Epidermal growth factor receptor tyrosine kinase inhibitors as a first-line therapy for never-smokers with adenocarcinoma of the lung having asymptomatic synchronous brain metastases. Lung Cancer. 2009;65(3):351-354.

38. Porta R, Sanchez-Torres JM, Paz-Ares L, et al. Brain metastases from lung cancer responding to erlotinib: the importance of EGFR mutation. Eur Respir J. 2011;37(3):624-631.

39. Ma S, Xu Y, Deng Q, et al. Treatment of brain metastases from nonsmall cell lung cancer with whole brain radiotherapy and gefitinib in a Chinese population. Lung Cancer. 2009;65(2):198-203.

40. Lind JS, Lagerwaard FJ, Smit EF, et al. Phase I study of concurrent whole brain radiotherapy and erlotinib for multiple brain metastases from non-small-cell lung cancer. Int J Radiat Oncol Biol Phys. 2009;74(5):1391-1396.

41. Olmez I, Donahue BR, Butler JS, et al. Clinical outcomes in extracranial tumor sites and unusual toxicities with concurrent whole brain radiation (WBRT) and erlotinib treatment in patients with non-small cell lung cancer (NSCLC) with brain metastases. Lung Cancer. 2010;70(2): 174-179.

42. Togashi Y, Masago K, Masuda S, et al. Cerebrospinal fluid concentration of gefitinib and erlotinib in patients with non-small cell lung cancer. Cancer Chemother Pharmacol. 2012;70(3):399-405.
43. Shi Y, Au JS, Thongprasert S, et al. A prospective, molecular epidemiology study of EGFR mutations in Asian patients with advanced non-small-cell lung cancer of adenocarcinoma histology (PIONEER). J Thorac Oncol. 2014;9(2):154-162.

44. Johnson BE, Kris MG, Berry LD, et al. A multicenter effort to identify driver mutations and employ targeted therapy in patients with lung adenocarcinomas: The Lung Cancer Mutation Consortium (LCMC). J Clin Oncol. 2013;31:8019.

45. Chinnaiyan P, Huang S, Vallabhaneni G, et al. Mechanisms of enhanced radiation response following epidermal growth factor receptor signaling inhibition by erlotinib (Tarceva). Cancer Res. 2005;65(8): 3328-3335.

46. Guangdong Association of Clinical Trials. Icotinib treat the patient with brain metastases epidermal growth factor receptor (EGFR) mutant non small cell lung cancer comparing with whole brain radiotherapy. Available from: http://www.clinicaltrials.gov/ct2/show/NCT01724801?term $=$ NCT01724801\&rank=1. NLM identifier: NCT01724801. Accessed August 6, 2014.

47. Hata A, Katakami N, Kaji R, et al. Erlotinib for whole-brain-radiotherapyrefractory leptomeningeal metastases after gefitinib failure in a lung adenocarcinoma patient. J Thorac Oncol. 2012;7(4):770-771.

48. Tetsumoto S, Osa A, Kijima T, et al. Two cases of leptomeningeal metastases from lung adenocarcinoma which progressed during gefitinib therapy but responded to erlotinib. Int J Clin Oncol. 2012;17(2): 155-159.
OncoTargets and Therapy

\section{Publish your work in this journal}

OncoTargets and Therapy is an international, peer-reviewed, open access journal focusing on the pathological basis of all cancers, potential targets for therapy and treatment protocols employed to improve the management of cancer patients. The journal also focuses on the impact of management programs and new therapeutic agents and protocols on

\section{Dovepress}

patient perspectives such as quality of life, adherence and satisfaction The manuscript management system is completely online and includes a very quick and fair peer-review system, which is all easy to use. Visit http://www.dovepress.com/testimonials.php to read real quotes from published authors. 